\title{
Importance of Physical Dispersion in Surface Wave and Free Oscillation Problems: Review
}

\author{
Hiroo Kanamori and Don L. Anderson
}

\author{
Seismological Laboratory, California Institute of Technology, Pasadena, California $91 / 25$
}

\begin{abstract}
Physical dispersion resulting from anelasticity is investigated from the point of view of linear viscoelastic models and causality relations. It is concluded that inasmuch as $Q$ in the earth's mantle is nearly independent of frequency, at least in the seismic frequency band, a dispersion relation in the form of $C(\omega)$ $=C\left(\omega_{r}\right)\left[1+\left(1 / \pi Q_{m}\right) \ln \left(\omega / \omega_{r}\right)\right]$ must be used for correcting the effect of physical dispersion arising from anelasticity. (Here $C(\omega)$ is the phase velocity of either body waves, surface waves, or free oscillations, $\omega$ is the angular frequency, $\omega_{r}$ is the reference angular frequency, and $Q_{m}$ is the path average $Q$ for body waves or $Q$ of a surface wave or a mode of angular frequency $\omega$; for surface waves and free oscillations, $C\left(\omega_{r}\right)$ should be understood as the phase velocity at $\omega$ computed by using the elastic moduli at $\omega=\omega_{r}$.) The values of $Q$ outside the seismic frequency band affect mainly the absolute value of the phase velocity but do not affect significantly the relative dispersion within the seismic frequency band. Even if the microscopic mechanism of attenuation is nonlinear, this dispersion relation can be used if departure from elasticity is relatively small, so that the signal can be approximated by a superposition of propagating harmonic waves. Since surface wave and free oscillation $Q$ is 100-500 for fundamental modes, a correction of $0.5-1.5 \%$ must be made for joint interpretation of body wave and surface wave data. This correction is nearly 1 order of magnitude larger than the uncertainties associated with these data and are therefore very significant. When this correction is made, the discrepancy between the observed surface wave phase velocities and free oscillation periods and those predicted by the Jeffreys or Gutenberg model becomes much smaller than has previously been considered.
\end{abstract}

\section{INTRODUCTION}

Anelasticity of the earth causes physical dispersion of seismic waves. While the importance of physical dispersion has long been recognized in seismic body wave studies, it has been either ignored or assumed to be negligible in most surface wave and free oscillation studies. The purpose of this paper is to clarify several points which are somewhat obscure in the literature on this subject. This obscurity appears to be partially responsible for the neglect of physical dispersion in surface wave and free oscillation studies. We will show that under conditions prevailing in the earth's interior, physical dispersion has a very significant effect on surface wave phase and group velocities and free oscillation periods. Although this effect has been discussed by Jeffreys [1965, 1967], Carpenter and Davies [1966], Davies [1967], Randall [1976], and, in more detail, Liu et al. [1976], we will look at the problem from a different point of view to emphasize further the importance of physical dispersion in surface wave and free oscillation studies.

A number of observations show that the intrinsic quality factor $Q$ in the earth's mantle depends very little upon frequency $f$ at least over the seismic frequency band from $1 \mathrm{~Hz}$ to 1 cycle/h [Knopoff, 1964]. Figure 1, which is constructed from Jackson and Anderson [1970], demonstrates this situation. Although a variation of a factor of 2 or 3 may exist, no obvious trend is seen, except that $Q_{\alpha}$ may increase at frequencies higher than about $1 \mathrm{~Hz}$. We accept this frequency independence of $Q$ over most of the seismic band and use it as the basis of the following discussion. It should be stressed, however, that the existence and importance of physical dispersion do not depend on the exact constancy of $Q$. As we will show, it is only where $Q$ varies as $f$ or $f^{-1}$ that one can argue that physical dispersion may be unimportant. In this situation, however, the $Q$ is in general very large, and the material is very nearly elastic. As we will show, the observed $Q$ of the earth is inconsistent with these requirements in both magnitude and frequency depen-

Copyright $\odot 1977$ by the American Geophysical Union.

Paper number 6R0680 dence. The earth therefore cannot be treated as an infinitesimal perturbation from a purely elastic body. Lack of appreciation of this point has led some authors to propose a nonlinear mechanism of attenuation. This is unnecessary. Laboratory data on metals, oxides, and salts over broad ranges of temperature, frequency, and attenuation are adequately explained with linear theories.

There appear to be three major reasons for the neglect of physical dispersion:

1. For a simple damped linear harmonic oscillator the inclusion of an infinitesimal attenuation $\epsilon$ changes the natural frequency of the system from $\omega$ to $\omega\left(1-C \epsilon^{2}\right)$, where $C$ is a constant. Since $\epsilon \sim Q^{-1}$, the effect can be ignored for $Q>100$, a typical value in the earth's mantle.

2. Knopoff and MacDonald [1958] showed that the inclusion of infinitesimal attenuation in a linear system results in $Q$ which is proportional to odd powers of frequency; therefore a constant $Q$ model (i.e., $Q \sim \omega^{\circ}$ ) is inconsistent with a linear system. This led Knopoff and MacDonald to introduce a

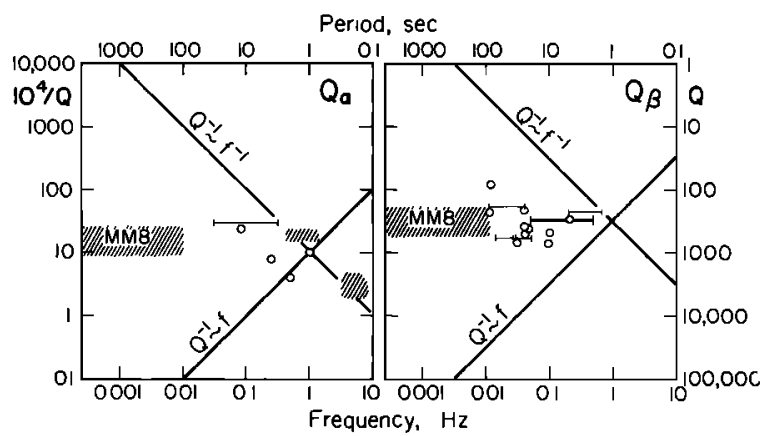

Fig. 1. Observed $Q$ for $P$ waves $\left(Q_{\alpha}\right)$ and $S$ waves $\left(Q_{\beta}\right)$ for the earth's mantle. The average values for the whole mantle are shown. For reference to individual data points, see Jackson and Anderson [1970]. The lines for $Q^{-1} \sim f^{-1}$ and $Q^{-1} \sim f$ are shown for reference. In calculating the average $Q$ for model MM8 [Anderson et al., 1965] a high- $Q$ lower mantle is assumed. 
nonlinear model. No explicit dispersion relation has been obtained for such a nonlinear system.

3. Futterman's [1962] dispersion theory, which is widely used in body wave seismology, predicts that inclusion of anelasticity increases the propagation velocity of a pulse in the medium. This apparent paradox has been challenged by some investigators [e.g., Stacey et al., 1975]. Because of this paradox, Futterman's [1962] theory, which is widely used in phase equalization of body waves, has not been extensively used in surface wave and free oscillation studies where the absolute phase velocity or period is the major concern.

We will show, using a linear model, that while all of the above arguments are correct, they do not directly apply to the situation in the earth's mantle. Lomnitz [1957] also showed from quite general considerations that dispersion must accompany absorption.

\section{Linear Viscoelastic Model}

The behavior of anelastic solids can be most conveniently described with a linear viscoelastic body. In the present discussion we follow the notation and the definition of Gross [1953].

For a purely elastic solid the stress $\sigma$ and strain $\alpha$ relation is given by $\sigma=E_{0} \alpha$, where $E_{0}$ is the elastic modulus. If we consider a step function application of strain $\alpha(t)=H(t)$ (where $H(t)$ is the Heaviside step function), the stress is given by $\sigma(t)=E_{0} H(t)$.

For a viscoelastic solid the response to a step strain application can be expressed as

$$
\sigma(t)=E_{0} H(t)+\Psi(t)
$$

where $\bar{\Psi}(t)$ is the relaxation function with $\bar{\Psi}(t)=0(t<0)$ and $\bar{\Psi}(\infty)=0$. The instantaneous response is given by $E_{\infty} \equiv E_{0}+$ $\bar{\Psi}(0)$, which is called the instantaneous elastic modulus (or unrelaxed elastic modulus).

For a viscoelastic solid with one relaxation mechanism such as one modeled by a parallel connection of a spring (constant $k_{1}$ ) and a spring (constant $k_{2}$ ) plus a dashpot (viscosity $\eta$ ) connected in series, $E_{0}=k_{1}$ and

$$
\Psi(t)=k_{2} e^{-t / \tau}
$$

where $\tau=\eta / k_{2}$ is the relaxation time constant.

For a harmonic strain, $\alpha \sim e^{i \omega t}$. The stress can be expressed by $\sigma=E^{*}(\omega) \alpha$, where

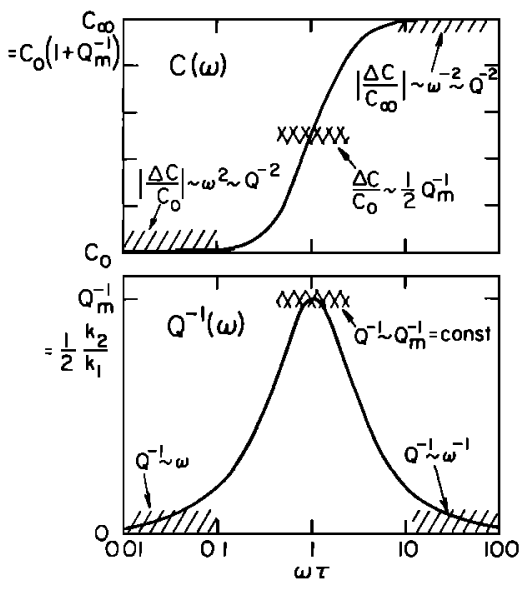

Fig. 2. The specific attenuation function $Q^{-1}(\omega)$ and the phase velocity $C(\omega)$ as a function of frequency for a linear viscoelastic model with a single relaxation mechanism.

$$
E^{*}(\omega)=\left[E_{0}+E_{1}(\omega)\right]+i E_{2}(\omega)
$$

is the complex elastic modulus. Since $\Psi(t)$ is the indicial response,

$$
E_{1}(\omega)+i E_{2}(\omega)=i \omega \int_{0}^{\infty} \bar{\Psi}(t) e^{-t \omega t} d t
$$

Consider a plane wave propagating in this medium:

$$
\exp [-\omega x / 2 Q(\omega) C(\omega)] \exp \{i \omega[t-x / C(\omega)]\}
$$

where $x$ is the distance, $C(\omega)$ the phase velocity, and $Q(\omega)$ the quality factor as defined in the usual manner. If we assume that the departure from elasticity is small, so that $\left|E_{0}\right| \gg$ $\left|E_{1}(\omega)\right|$ and $\left|E_{0}\right| \gg\left|E_{2}(\omega)\right|$, then it is easy to show that

$$
C(\omega)=\left\{\left[E_{0}+E_{1}(\omega)\right] / \rho\right\}^{1 / 2}
$$

and

$$
Q^{-1}(\omega)=E_{2}(\omega) / E_{0}
$$

where $\rho$ is the density. For the spring-dashpot system discussed above, (1), (2), (3), and (4) immediately give

$$
C(\omega)=C_{0}\left[1+\frac{1}{2} \frac{k_{2}}{k_{1}} \frac{(\omega \tau)^{2}}{1+(\omega \tau)^{2}}\right]
$$

where $C_{0}=\left(k_{1} / \rho\right)^{1 / 2}$, and

$$
Q^{-1}(\omega)=\frac{k_{2}}{k_{1}} \frac{\omega \tau}{1+(\omega \tau)^{2}}
$$

which give the familiar attenuation and dispersion relations as schematically shown in Figure 2. $Q^{-1}(\omega)$ becomes maximum at $\omega=1 / \tau$ (absorption peak), where $Q^{-1}(\omega)=k_{2} / 2 k_{1} \equiv Q_{m}{ }^{-1}$. The low- and high-frequency limits of the phase velocity are $C_{0}$ and $C_{\infty}=C_{0}\left[1+\frac{1}{2}\left(k_{2} / k_{1}\right)\right]=C_{0}\left(1+Q_{m}{ }^{-1}\right)$, the difference being $C_{0} Q_{m}{ }^{-1}$. Since $Q^{-1}=0$ corresponds to the purely elastic state, an infinitesimal departure from elasticity corresponds to the ranges where $\omega \tau \gg 1$ or $\omega \tau \ll 1$ (hatching in Figure 2). It is evident from (5) and (6) that when $\omega \tau \ll 1, Q^{-1}(\omega) \sim$ $\left(k_{2} / k_{1}\right) \omega \tau$ and $C(\omega) \sim C_{0}\left[1+\frac{1}{2}\left(k_{2} / k_{1}\right)(\omega \tau)^{2}\right]$, and therefore $C(\omega) \sim C_{0}\left[1+\frac{1}{2}\left(k_{1} / k_{8}\right) Q^{-2}\right]$. Similarly, if $\omega \tau \gg 1, Q^{-1}(\omega) \sim$ $\left(k_{2} / k_{1}\right)(1 / \omega \tau)$ and

$$
C(\omega) \sim C_{\infty}\left[1-\frac{k_{2}}{2 k_{1}+k_{2}} \frac{1}{(\omega \tau)^{2}}\right]
$$

and therefore

$$
C(\omega) \sim C_{\infty}\left[1 \frac{k_{1}{ }^{2}}{\left(2 k_{1}+k_{2}\right) k_{2}} Q^{-2}\right]
$$

Tisus in these limits the fractional change of the phase velocity is proportional to $Q^{-2}$ (i.e., the $Q$ effect is only second order). This is the generalization of point 1 discussed in the introduction. For $Q \geq 100$, typical values for the earth's mantle, the physical dispersion is unimportant in this case. However, in this case, $Q^{-1}$ itself has either $\omega$ or $1 / \omega$ dependence. As is clearly shown in Figure 1, $Q^{-1}(\omega)$ for the earth's mantle does not show such dependence, except possibly for $P$ waves at high frequencies. Therefore the argument made in point 1 in the introduction is not relevant to the problem of the earth's interior, at least over most of the seismic frequency band.

In the linear viscoelastic model shown in Figure 2, $Q^{-1}(\omega)$ is nearly constant when $\omega \sim 1 / \tau$. This situation is closer to that of the earth's mantle, although $Q^{-1}(\omega)$ of the earth has a much broader peak. This range of $\omega$ corresponds to the region 
(cross-hatching in Figure 2) where departure from pure elasticity can no longer be regarded as 'infinitesimal.' In this range, $Q^{-1}(\omega) \sim \frac{1}{2}\left(k_{2} / k_{1}\right) \equiv Q_{m}^{-1}=$ const, and the fractional change of the phase velocity from that of the purely elastic states is $\mathfrak{l}\left(k_{2} / k_{1}\right) \sim 1 Q_{m}^{-1}$. Thus the effect of $Q$ on the phase velocity becomes first order. For $Q_{m} \sim 100$ the phase velocity changes by as much as $0.5 \%$. Since the actual $Q^{-1}(\omega)$ function for the earth is considerably different from (6), the above argument is valid only qualitatively. However, it is important to note that to obtain a nearly frequency-independent $Q$ requires departure from elasticity beyond the infinitesimal range, so that inferences from infinitesimal theories such as points 1 and 2 mentioned in the introduction are not relevant to the problems of the earth's mantle. When $Q$ is constant or nearly so, the fractional change in the phase velocity is proportional to $Q^{-1}$ rather than $Q^{-2}$ predicted by infinitesimal theories. This is implicit in the work of Lomnitz [1957], whose model gives a slightly frequency-dependent $Q$.

\section{Constant $Q$ Model}

Various attempts have been made to explain the nearly constant $Q$ from at least 1 -s to 1 -hour periods (Figure 1 ) for the earth's mantle [Knopoff and MacDonald, 1958; Lomnitz, 1957; Savage. 1965; Mason, 1969; Liu et al., 1976]. Liu et al. showed that it is possible to construct a band-limited constant $Q$ model by using a linear viscoelastic model. For purposes of the present discussion it is most convenient to use such a model.

As was shown earlier, a departure from elasticity can be characterized by a relaxation function $\Psi(t)$. This function can be expressed as a superposition of the elementary relaxation function $e^{-s t}$ which corresponds to the simple spring-dashpot system (equation (1)). Thus

$$
\Psi(t)=\int_{0}^{\infty} \tilde{N}(s) e^{-s t} d s
$$

where $s=1 / \tau$ is the relaxation frequency and $\bar{N}(s)$ is the relaxation spectral density (see also Gross [1953]). Combining this with (2), we have

$$
E_{1}(\omega)=\int_{0}^{\infty} \frac{\omega^{2}}{s^{2}+\omega^{2}} \bar{N}(s) d s
$$

and

$$
E_{2}(\omega)=\int_{0}^{\infty} \frac{s \omega}{s^{2}+\omega^{2}} \bar{N}(s) d s
$$

One of the simplest ways of constructing a band-limited constant $Q$ model is to introduce a relaxation spectrum $\bar{N}(s)$ in the form

$$
\begin{array}{cl}
\vec{N}(s)=A / s & s_{1}<s<s_{2} \\
\bar{N}(s)=0 & \text { elsewhere }
\end{array}
$$

where $A$ is a constant.

Carrying out integrations (8) and (9) and using (3) and (4), we have

$$
Q^{-1}(\omega)=\frac{2}{\pi Q_{m}} \tan ^{-1}\left[\frac{\omega\left(s_{2}-s_{1}\right)}{\omega^{2}+s_{1} s_{2}}\right]
$$

and

$$
C(\omega)=C_{0}\left[1+\frac{1}{2 \pi Q_{m}} \ln \frac{s_{2}^{2}\left(s_{1}{ }^{2}+\omega^{2}\right)}{s_{1}^{2}\left(s_{2}{ }^{2}+\omega^{2}\right)}\right]
$$

where $Q_{m}^{-1}$ is the maximum of $Q^{-1}(\omega)\left(Q_{m}^{-1}=(\pi / 2)\left(A / E_{0}\right)\right)$.
These relations are plotted in Figure 3. If we arbitrarily choose a very small $s_{1}$ and a very large $s_{2}$, then for $s_{1} \ll \omega \ll s_{2}$ we have

$$
Q^{-1}(\omega) \sim Q_{m}^{-1}=\text { const }
$$

and

$$
C(\omega)=C_{0}\left[1+\frac{1}{\pi Q_{m}} \ln \left(\frac{\omega}{s_{1}}\right)\right]
$$

or

$$
C(\omega)=C_{\infty}\left[1-\frac{1}{\pi Q_{m}} \ln \left(\frac{s_{2}}{\omega}\right)\right]
$$

where

$$
C_{\infty}=C_{0}\left[1+\frac{1}{\pi Q_{m}} \ln \left(\frac{s_{2}}{s_{1}}\right)\right]
$$

and $\left(1 / \pi Q_{m}\right) \ln \left(s_{2} / s_{1}\right) \ll 1$ is assumed. These relations are fundamentally identical to those derived by various investigators [e.g., Kolsky, 1956; Lomnitz, 1957; Futterman. 1962; Savage, 1965; Strick, 1967; Liu et al., 1976] on the basis of a variety of assumptions, methods, and approximations and can be regarded as a universal dispersion relation for any linear models in the frequency range where $Q$ is constant. They can also be derived from the equations given by Nowick and Berry [1961] for a log normal distribution of relaxation times. We choose $s_{1}$ and $s_{2}$ in such a way that the frequency range of our interest, say $1 \mathrm{~Hz}$ to $\mathrm{l}$ cycle/h, is completely bracketed by $s_{1}$ and $s_{2}$. It is important to note that while the ratio $C_{\infty} / C_{0}=$ $\left[1+\left(1 / \pi Q_{m}\right) \ln \left(s_{2} / s_{1}\right)\right]$ depends upon the ratio of the arbitrary upper and lower bounds of the angular frequency $s_{2} / s_{1}$, the ratio of the phase velocities at angular frequencies $\omega_{1}$ and $\omega_{2}$ within the range of our interest $\left(s_{1} \ll \omega_{1}<\omega_{2} \ll s_{2}\right)$

$$
\frac{C\left(\omega_{2}\right)}{C\left(\omega_{1}\right)} \sim 1+\frac{1}{\pi Q_{m}} \ln \left(\frac{\omega_{2}}{\omega_{1}}\right)
$$

does not depend upon these arbitrary constants. Thus in the frequency range where $Q=Q_{m}=$ const, the relative dispersion can be determined unambiguously without knowledge of $Q$ and the phase velocity outside the frequency band considered. This conclusion is based on the linear model considered above,
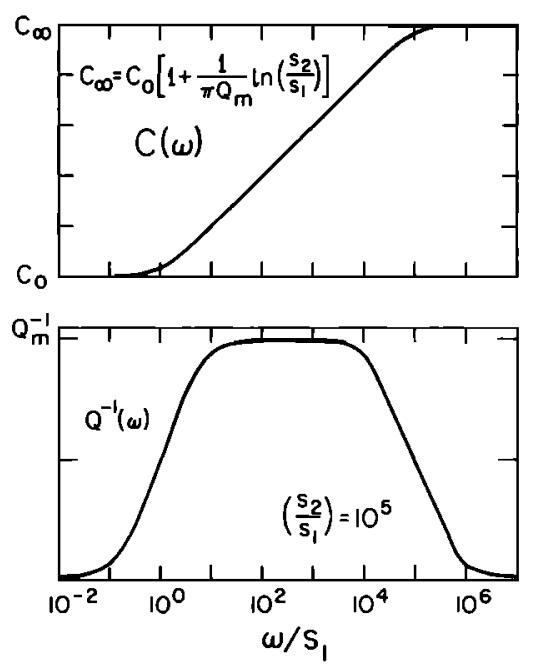

Fig. 3. Band-limited constant $Q$ model derived from a linear viscoelastic model. Variables $s_{1}$ and $s_{2}$ are low-frequency and high-frequency cutof, respectively. The curves are computed for $s_{2} / s_{1}=10^{5}$. 
and in more general cases the relative dispersion relation can be affected by $Q$ outside the range $\omega_{1}<\omega<\omega_{2}$. This effect will be discussed in a later section.

The necessity for introducing a spectrum of relaxation times or a superposition of absorption mechanisms is not unique to seismology. Laboratory measurements of attenuation, even on relatively pure materials, usually show a broader absorption peak than can be explained by a single relaxation time. A different relaxation spectrum, a log normal distribution, has been studied at length [Nowick and Berry. 1961] to explain broad regions of nearly constant $Q$ in metals.

The relaxation function for the model introduced in this section can be obtained by using (7) and (10):

$$
\Psi(t)=\frac{2 E_{0}}{\pi Q_{m}} \int_{s_{1}}^{s_{2}} \frac{1}{s} e^{-s t} d s=\frac{2 E_{0}}{\pi Q_{m}}\left[E i\left(-s_{2} t\right)-E i\left(-s_{1} t\right)\right]
$$

where $E i(-x)$ is the exponential integral defined by

$$
\begin{array}{r}
E i(-x)=-\int_{x}^{\infty} \frac{e^{-t}}{t} d t=\ln x+\gamma-x+\frac{x^{2}}{2 \cdot 2 !}-\cdots \\
+\frac{(-x)^{r}}{r \cdot r !}+\cdots \quad(x>0)
\end{array}
$$

(see, e.g., Jahnke and Emde [1945]). When $s_{2} \gg s_{1}$, we have

$$
\begin{aligned}
& \Psi(t)=\frac{2 E_{0}}{\pi Q_{m}}\left[\ln \left(\frac{s_{2}}{s_{1}}\right)-\left(s_{2}-s_{1}\right) t\right] \quad t \ll s_{2}^{-1} \\
& \Psi(t)=\frac{2 E_{0}}{\pi Q_{m}}\left\{\ln \left(\frac{s_{2}}{s_{1}}\right)-\left[-E i\left(-s_{2} t\right)+\ln s_{2} t+\gamma\right]\right\} \\
& \Psi(t)=-\frac{2 E_{0}}{\pi Q_{m}} E i\left(-s_{1} t\right) \quad t \sim s_{2}^{-1} \\
& \Psi(t)=0 \quad t \gg s_{1}^{-1}
\end{aligned}
$$

\section{RELATION to Lomnitz's RELATION}

Lomnitz [1957] introduced a logarithmic creep function of the form

$$
\Psi(t)=q \ln (1+a t)
$$

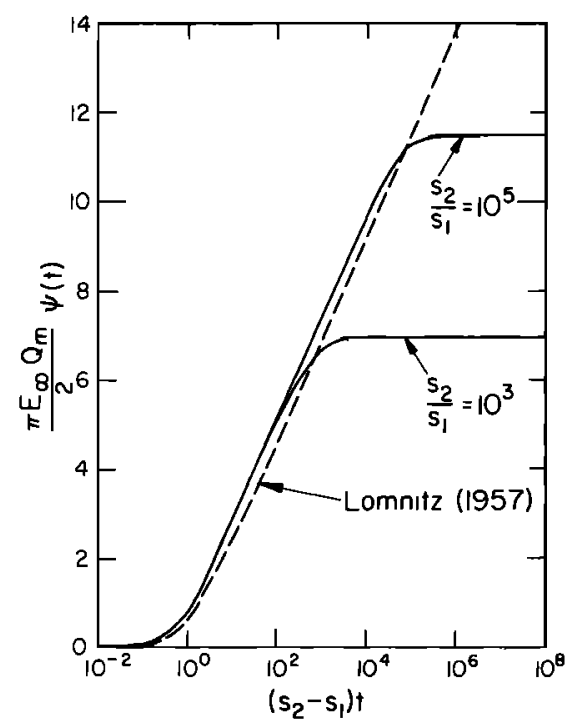

Fig. 4. Creep functions $\Psi(t)$ for the Lomnitz [1957] model and the band-limited constant $Q$ model. to construct a constant $Q$ model. (Lomnitz used $\phi(t)$ for the creep function; here it is replaced by $\Psi(t)$ in following the notation of Gross [1953].) Although this creep function does not pass in the limit to the static case (i.e., $\Psi(t) \rightarrow \infty$ when $t \rightarrow$ $\infty$ [Kogan, 1966]), it describes the anelastic behavior of solids reasonably well. It is instructive to compare the creep function for the model introduced in the previous section with Lomnitz's logarithmic creep function.

The creep function $\Psi(t)$ is defined by

$$
\alpha(t)=\left(1 / E_{\infty}\right)+\Psi(t)
$$

where $\alpha(t)$ is the change in the strain after application of a step function stress $\sigma(t)=H(t), E_{\mathrm{m}}$ is the unrelaxed elastic modulus, and $\Psi(t)=0(t \leq 0)$ [Gross, 1953, equation (1)].

In the preceding section, only the relaxation function $\Psi(t)$ was obtained (equation (16)). In general, the creep function $\Psi(t)$ is related to the relaxation function through the Volterra integral equation [Gross, 1953, equation (58)], but it is not always easy to obtain the creep function from the relaxation function. However, when the departure from elasticity is small, it can be obtained by using relations (36) and (40) of Gross [1953]. The result is

$$
\frac{d \Psi}{d t}=\frac{2}{\pi E_{\infty} Q_{m}} \int_{s_{1}}^{s_{2}} e^{-t s} d s=-\frac{2}{\pi E_{\infty} Q_{m}} \frac{1}{t}\left[e^{-s_{2} t}-e^{-s_{1} t}\right]
$$

therefore

$$
\begin{aligned}
& \Psi(t)=-\frac{2}{\pi E_{\infty} Q_{m}}\left[\int_{0}^{t} \frac{e^{-s_{2} t}}{t} d t-\int_{0}^{t} \frac{e^{-s_{1} t}}{t} d t\right] \\
& \Psi(t)=\frac{2}{\pi E_{\infty} Q_{m}}\left\{\ln \left(\frac{s_{2}}{s_{1}}\right)-\left[E i\left(-s_{2} t\right)-E i\left(-s_{1} t\right)\right]\right\} \\
& \Psi(t)=\frac{2}{\pi E_{\infty} Q_{m}} \ln \left(\frac{s_{2}}{s_{1}}\right)-\frac{1}{E_{0} E_{\infty}} \Psi(t)
\end{aligned}
$$

By using (17) we find

$$
\Psi(\infty)=\frac{2}{\pi E_{\infty} Q_{m}} \ln \left(\frac{s_{2}}{s_{1}}\right)
$$

and for very small $t$,

$$
\Psi(t)=\frac{2}{\pi E_{\infty} Q_{m}}\left(s_{2}-s_{1}\right) t
$$

In order to compare this with Lomnitz's relation (19), $\Psi(t)=q$ In $(1+a t)$, we set $q=\left(2 / \pi E_{\infty} Q_{m}\right)$ and $a=\left(s_{2}-s_{1}\right) \sim s_{2}$. These relations are plotted in Figure 4 as a function of $\left(s_{2}-s_{1}\right) t$ for two cases, $s_{2} / s_{1}=10^{5}$ and $s_{2} / s_{1}=10^{3}$. The creep function (21) is very similar to Lomnitz's logarithmic creep function for $0<$ $t<s_{1}{ }^{-1}$ and approaches the static limit $\left(2 / \pi E_{\odot} Q_{m}\right) \ln \left(s_{2} / s_{1}\right)$ at $t \rightarrow \infty$, the difficulty of Lomnitz's [1957] creep function at $t \rightarrow$ $\infty$ thereby being removed.

\section{Relation to Futterman's Dispersion Relation}

Futterman's [1962] relation was derived from the KramersKrönig causality relations together with the condition that the phase velocity approaches $C_{0}$ (denoted as $C$ in the paper by Futterman [1962]) in the low-frequency limit. His relation gives higher phase and group velocities in an attenuating medium than in a nonattenuating medium. This apparent paradox appears to have partially hindered the use of this dispersion relation in surface wave and free oscillation studies where the absolute arrival time is the major concern. We will show that this paradox does not constitute a real physical implausibility but is a result of the arbitrary assumption that 
$C_{0}$ is the reference elastic velocity. This point was recently discussed by Savage [1976].

The Kramers-Krönig causality relations result from the physical requirement that the response of a linear system to a transient input should not precede the time of the application of the input, say $t_{0}$. It is important to note that the causality relations alone do not specify when the response should start; it can start at any time after $t_{0}$. In terms of the linear viscoelastic model discussed in the preceding section this situation corresponds to specifying only the relative dispersion relation but not the absolute velocity. In other words, the causality relations determine a group of curves having a common relative dispersion relation but not a single curve. In order to determine a unique dispersion curve a reference velocity must be set at a certain frequency $\omega=\omega_{r}$. Suppose we consider a group of linear viscoelastic models which have a common $Q_{m}{ }^{-1}, s_{1}$, and $s_{2}$ but different $C_{\infty}$ (phase velocity at infinite frequency). Curves 1 and 2 in Figure 5 show the dispersion curves for two of these models. Since they are solutions of the viscoelastic model, both curve 1 and curve 2 must satisfy the causality relations. However, the starting time of the response is obviously different between them. We next consider another set having a common $Q_{m}{ }^{\prime-1}$ which is smaller than $Q_{m}{ }^{-1}, s_{1}$ and $s_{2}$ being kept unchanged. Curves $1^{\prime}$ and $2^{\prime}$ in Figure 5 show two models of this group. If we choose $\omega_{r}$ at 0 and compare curves 1 and $1^{\prime}$, it is obvious that the model with $Q_{m}$ (more attenuating) has a higher phase velocity than the model with $Q_{m}{ }^{\prime}$ (less attenuating) at any frequency. This situation corresponds to Futterman's [1962] model. On the other hand, if we choose $\omega_{r}$ at infinity and compare curves 2 and $2^{\prime}$, then it is clear that the high- $Q$ model has a higher phase velocity than the low- $Q$ model at any frequency. Thus the paradox of Futterman's model is simply a result of an arbitrary choice of the reference frequency and does not represent a fundamental physical defect of the model.

Although the material behaves elastically at both very high and very low frequencies, since $Q^{-1} \rightarrow 0$, the response at high frequencies represents the instantaneous response and therefore corresponds to the more common interpretation of elastic behavior. Also, since ideal elastic behavior is only approached at very low temperatures, the correspondence between temperature and frequency for activated processes indicates that the high-frequency limit should be taken as the elastic reference value. The activated process assumption is consistent with our simple model, since the viscosity of the dashpot varies exponentially as temperature. Of course at absolute zero the viscosity is infinite, and the high- and low-frequency elastic properties are the same. However, an increase in temperature

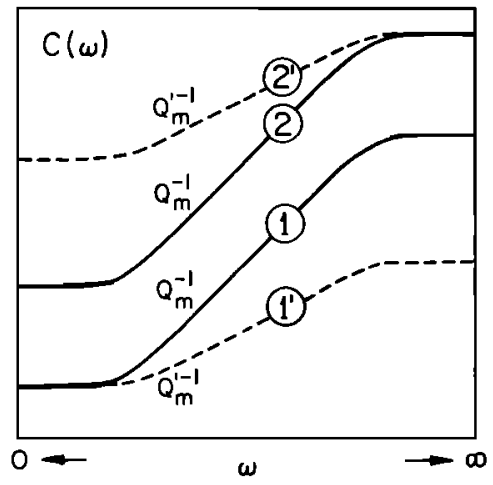

Fig. 5. Phase velocity curves for different values of $Q$ and reference velocity. will leave the high-frequency limit unchanged, since the dashpot does not have time to respond, but the system is less stiff for low frequencies, and therefore the velocity decreases.

\section{Effect of $Q$ Outside the Seismic Frequency Band}

We have shown that a constant $Q$ over the seismic frequency band can be modeled with a linear viscoelastic solid. In this model, $Q^{-1}(\omega)$ falls off to zero outside the seismic frequency band. However, the value of $Q$ outside the range from $1 \mathrm{~s}$ to 1 hour is not very well known for the earth. It is possible that $Q^{-1}(\omega)$ in the earth's mantle outside the seismic frequency band is very different from that of linear models. A question then arises concerning what effect such a variation of $Q$ has on the dispersion relation in the seismic band. In order to answer this question it is convenient to use the Kramers-Krönig causality relations. Following Lamb [1962], we consider a propagating harmonic plane wave

$$
f(x, t) \sim \exp \left\{i \omega\left[t-\frac{n(\omega)}{C_{\omega}} x\right]\right\}
$$

where $n(\omega)$ is the complex refraction index

$$
n(\omega)=n_{1}(\omega)-i n_{2}(\omega)
$$

with $n_{1}(\infty)=1$ and $n_{2}(\infty)=0$. $C_{\infty}$ is the phase velocity at $\omega=$ $\infty$, which is chosen as the reference frequency. Since $n(\omega)$ must satisfy $n(-\omega)=n^{*}(\omega)$ (asterisk denotes the complex conjugate), $n_{1}(-\omega)=n_{1}(\omega)$ and $n_{2}(-\omega)=-n_{2}(\omega)$.

The phase velocity $C(\omega)$ and $Q^{-1}(\omega)$ can then be written as

$$
C(\omega)=C_{\infty} / n_{1}(\omega)
$$

and

$$
Q^{-1}(\omega)=2 n_{2}(\omega)
$$

The causality condition requires that $n(\omega)$ be analytic in the lower half of the complex $\omega$ plane. This analyticity condition is then expressed in terms of the Kramers-Krönig relations (see, for example, Morse and Feshbach [1953, p. 372]):

$$
\begin{aligned}
& n_{1}(\omega)=1+\frac{1}{\pi} P \int_{-\infty}^{+\infty} \frac{n_{2}\left(\omega^{\prime}\right)}{\omega^{\prime}-\omega} d \omega^{\prime} \\
& n_{2}(\omega)=-\frac{1}{\pi} P \int_{-\infty}^{+\infty} \frac{n_{1}\left(\omega^{\prime}\right)}{\omega^{\prime}-\omega} d \omega^{\prime}
\end{aligned}
$$

where $P$ denotes the Cauchy principal value. First we consider the simplest case (Figure 6) where

$$
\begin{gathered}
Q^{-1}(\omega)=\operatorname{sgn}(\omega) Q_{m}^{-1} \quad s_{1} \leq|\omega| \leq s_{2} \\
Q^{-1}(\omega)=0 \quad \text { elsewhere }
\end{gathered}
$$

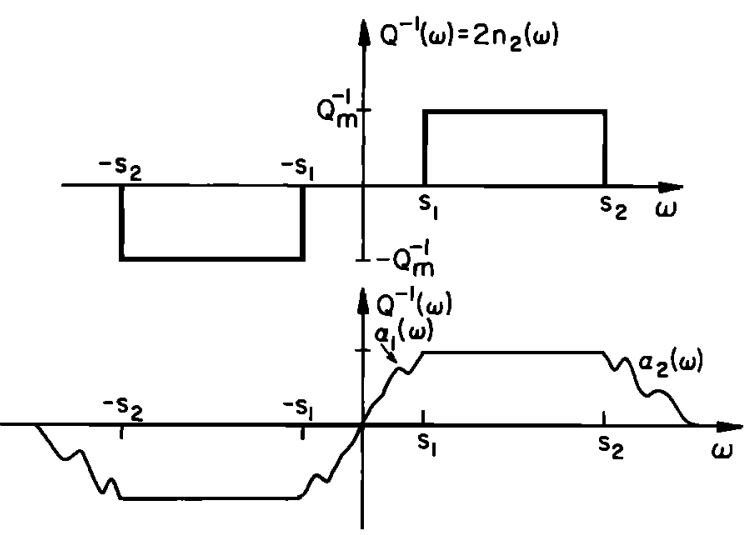

Fig. 6. Two models of $Q^{-1}(\omega)$ as a function of $\omega$. 
It is implied that the seismic frequency band is bracketed well within the range $s_{1} \leq \omega \leq s_{2}$. Carrying out integration (28) and using (26), we have

$$
\begin{aligned}
& \qquad C(\omega)=C_{\infty}\left[1-\left(2 \pi Q_{m}\right)^{-1} \ln \left|\frac{\omega^{2}-s_{1}^{2}}{s_{2}^{2}-\omega^{2}}\right|\right]^{-1} \\
& \text { For } s_{1} \ll \omega \ll s_{2}, \\
& C(\omega)=C_{\infty}\left[1+\left(\pi Q_{m}\right)^{-1} \ln \left(s_{2} / \omega\right)\right]^{-1} \sim \\
& C_{\infty}\left[1-\left(\pi Q_{m}\right)^{-1} \ln \left(s_{2} / \omega\right)\right]
\end{aligned}
$$

which is identical to (14) derived from the linear model.

We now introduce arbitrary variations of $Q^{-1}(\omega)$ outside the range $s_{1} \leq \omega \leq s_{2}$. We put

$$
\begin{aligned}
& Q^{-1}(\omega)=\alpha_{1}(\omega) \quad 0<|\omega|<s_{1} \\
& Q^{-1}(\omega)=\operatorname{sgn}(\omega) Q_{m}^{-1} \quad s_{1} \leq|\omega| \leq s_{2} \\
& Q^{-1}(\omega)=\alpha_{2}(\omega) \quad|\omega|>s_{2}
\end{aligned}
$$

where $\alpha_{\imath}(-\omega)=-\alpha_{i}(\omega)(i=1,2)$. Then by using (28) we have for $s_{1} \ll \omega \ll s_{2}$

$$
n_{1}(\omega)=\left[1-\left(\pi Q_{m}\right)^{-1} \ln \left(\omega / s_{2}\right)\right]+I_{1}+I_{2}
$$

where

$$
I_{1}=2 \int_{0}^{s_{1}} \frac{\alpha_{1}\left(\omega^{\prime}\right)}{\omega^{\prime 2}-\omega^{2}} \omega^{\prime} d \omega^{\prime}
$$

and

$$
I_{2}=2 \int_{s_{2}}^{\infty} \frac{\alpha_{2}\left(\omega^{\prime}\right)}{\omega^{\prime 2}-\omega^{2}} \omega^{\prime} d \omega^{\prime}
$$

Since $\omega \ll s_{2}$,

$$
I_{2} \sim 2 \int_{s_{2}}^{\infty} \frac{\alpha_{2}\left(\omega^{\prime}\right)}{\omega^{\prime}} d \omega^{\prime}
$$

which is a function of $s_{2}$ only and does not depend on $\omega$. This term therefore affects only the absolute value of the phase

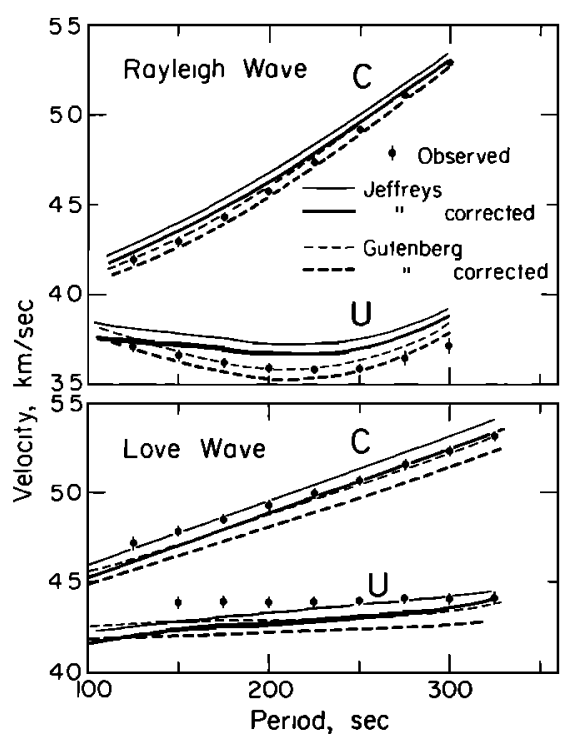

Fig. 7. Rayleigh wave and Love wave dispersion curves for uncorrected and corrected Jeffreys and Gutenberg models. The data points are taken from Kanamori [1970]. The error bars represent the standard deviation. velocity but not the relative dispersion relation. For the first integral, since $\omega \gg s_{1}$, we have

$$
\left|I_{1}\right| \sim 2 \frac{1}{\omega^{2}} \int_{0}^{s_{1}} \alpha_{1}\left(\omega^{\prime}\right) \omega^{\prime} d \omega^{\prime}<\left(\frac{s_{1}}{\omega}\right)_{\max 2}\left|\alpha_{1}(\omega)\right|
$$

Since $\omega \gg s_{1}$, this term is small unless $\left|\alpha_{1}(\omega)\right|$ becomes very large, i.e., very large absorption at low frequencies.

Thus we conclude that if the value of $Q^{-1}(\omega)$ is moderate outside the seismic frequency band, its effect on the relative dispersion in the seismic frequency band can be ignored. In other words, the knowledge of $Q^{-1}(\omega)$ in the seismic frequency band is sufficient to evaluate the relative dispersion of seismic signals.

Strictly speaking, the above derivation is valid only for a linear system in which the propagation of a signal can be expressed as a linear superposition of plane waves in the form of (16). There is no evidence that the value of $Q$ in the earth's mantle depends on the wave amplitude. Also, the similarity of wave forms between near-vertical multiple $S c S$ ( $S$ wave reflected from the core-mantle boundary) phases strongly suggests that no drastic distortion of wave form, other than the exponential decay, occurs during propagation. These observations suggest that even if the microscopic mechanism of attenuation is nonlinear, the propagation of the signal can be approximated by a superposition of plane waves in the form of (16), and therefore the dispersion relation (15) holds with good approximation.

Thus inasmuch as $Q^{-1}(\omega)$ is nearly constant in the seismic frequency band, the conclusion that seismic signals should exhibit a physical dispersion in the form of (15) appears inescapable. It should be repeated that an expression identical to (15) can also be derived from the equations of Lomnitz [1957], Futterman [1962], and Nowick and Berry [1961] and is a quite general description of the dispersion that must accompany absorption in linear systems.

\section{Significance of Physical Dispersion in Seismology}

The effect of physical dispersion is most important in the inversion of body wave, surface wave, and free oscillation data. Since this problem has already been discussed in detail by Anderson et al. [1976], Hart et al. [1976], and Anderson and Hart [1976b], we limit our discussion to some points of historical interest.

In the late 1950's to early 1960's the first accurate measurements of long-period mantle surface waves and free oscillations were made, and introduction of digital computers made it possible to compute dispersion curves and free oscillation periods for various earth models. A significant difference was then discovered between the observed velocities or periods and those computed for the classic Jeffreys and Gutenberg models (Figures 7 and 8 ). Attempts to remove this discrepancy resulted in the later development of geophysical inversion theories. However, these classic models were based on short-period body waves, and therefore they should not be compared directly with the long-period surface wave and free oscillation data. It is necessary to apply corrections resulting from physical dispersion to the phase velocities or periods calculated for structures determined with body waves. Carpenter and Davies [1966] and Davies [1967] pointed out the importance of physical dispersion in surface wave problems and discussed the compatibility of surface wave and body wave data. Since detailed discussions are made in Liu et al. [1976], only a brief account is made in the following discussion. 


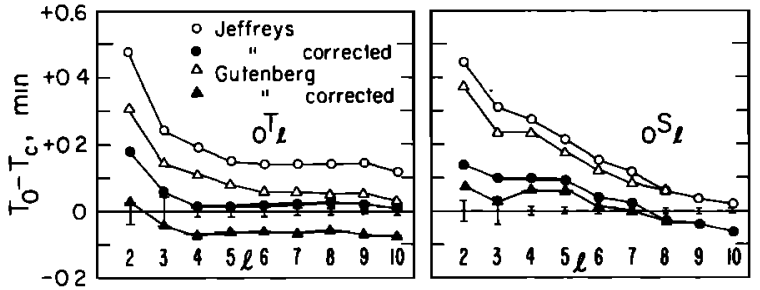

Fig. 8. Observed minus computed free oscillation periods for fundamental spheroidal and torsional modes. The observed values and error bars are taken from Table 2 of Anderson and Hart [1976a]. The Bullen A density model is used in the calculations.

Consider a $\mathrm{N}$-layered spherical earth model. In each layer the dispersion relations are given by

$$
\begin{aligned}
& \alpha_{l}(\omega)=\alpha_{l}\left(\omega_{r}\right)\left[1+\frac{1}{\pi Q_{\alpha l}} \ln \left(\frac{\omega}{\omega_{r}}\right)\right] \\
& \beta_{l}(\omega)=\beta_{l}\left(\omega_{r}\right)\left[1+\frac{1}{\pi Q_{\beta l}} \ln \left(\frac{\omega}{\omega_{r}}\right)\right]
\end{aligned}
$$

where $\alpha, \beta, Q_{\alpha}$, and $Q_{\beta}$ are $P$ velocity, $S$ velocity, $Q$ for $P$ waves and $Q$ for $S$ waves, respectively, $l$ is the layer index, and $\omega_{r}$ is the reference frequency taken at a body wave frequency.

Then the phase velocity of Love and Rayleigh waves (or torsional and spheroidal modes) at frequency $\omega$ computed for a structure defined at a body wave frequency can be corrected at each frequency by the following relations [Liu et al., 1976]:

$$
\begin{gathered}
\Delta C_{L}(\omega)=\sum_{l=1}^{N} \frac{\partial C_{L}(\omega)}{\partial \beta_{l}(\omega)} \Delta \beta_{l}(\omega) \\
=C_{L}(\omega)\left[\frac{1}{\pi} \ln \left(\frac{\omega}{\omega_{r}}\right)\right] \sum_{l=1}^{N} \frac{\beta_{l}}{C_{L}} \frac{\partial C_{L}}{\partial \beta_{l}} Q_{\beta l}{ }^{-1} \\
\Delta C_{L}(\omega)=C_{L}(\omega)\left[\frac{1}{\pi} \ln \left(\frac{\omega}{\omega_{r}}\right) Q_{L}^{-1}(\omega)\right] \\
\Delta C_{R}(\omega)=\sum_{l=1}^{N}\left[\frac{\partial C_{R}(\omega)}{\partial \beta_{l}(\omega)} \Delta \beta_{l}(\omega)+\frac{\partial C_{R}(\omega)}{\partial \alpha_{l}(\omega)} \Delta \alpha_{l}(\omega)\right] \\
\Delta C_{R}(\omega)=C_{R}(\omega)\left[\frac{1}{\pi} \ln \left(\frac{\omega}{\omega_{r}}\right)\right] \\
\cdot \sum_{l=1}^{N}\left[\frac{\beta_{l}}{C_{R}} \frac{\partial C_{R}}{\partial \beta_{l}} Q_{\beta l}^{-1}+\frac{\alpha_{l}}{C_{R}} \frac{\partial C_{R}}{\partial \alpha_{l}} Q_{\alpha l}{ }^{-1}\right] \\
\Delta C_{R}(\omega)=C_{R}(\omega)\left[\frac{1}{\pi} \ln \left(\frac{\omega}{\omega_{r}}\right) Q_{R}^{-1}(\omega)\right]
\end{gathered}
$$

where $Q_{L}(\omega)$ and $Q_{R}(\omega)$ are $Q$ for Love and Rayleigh waves. These corrections can be calculated by using either observed $Q_{L}(\omega)$ and $Q_{R}(\omega)$ (through (31) and (33)) or a given depth distribution of $Q_{\alpha}$ and $Q_{\beta}$ (through (30) and (32)). These corrections have been computed by Liu et al. [1976] for fundamental modes and by Hart et al. [1976] for overtones. In these calculations, $\omega_{r} / 2 \pi=1 \mathrm{~Hz}(1 \mathrm{~s})$.

Corrections for group velocity can be calculated from $\Delta C(\omega)$ by

$$
\frac{\Delta U}{U}=\frac{U}{C^{2}}\left(\frac{2 C}{U}-1\right) \Delta C+\frac{U}{C^{2}} \omega \frac{d(\Delta C)}{d \omega}
$$

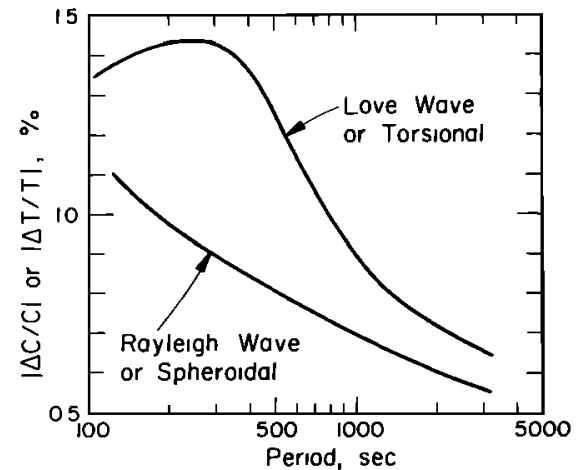

Fig. 9. Fractional change in Love wave (torsional mode) and Rayleigh wave (spheroidal mode) phase velocities (periods) as a function of period computed from the observed $Q$.

As has been shown by Liu et al. [1976] and Hart et al. [1976], these corrections amount to $0.5-1.5 \%$ (Figure 9). Since the standard deviation of the measurement of phase velocities and free oscillation periods is about $0.05-0.4 \%$ (see Figures 7 and 8 ), the above corrections are very significant. Although the details of the depth distribution of $Q_{\alpha}$ and $Q_{\beta}$ are not very well known at present, the values of $Q$ for Love and Rayleigh waves are fairly well established experimentally, at least over a period range from 100 to $1000 \mathrm{~s}$. Thus we believe that the corrections for this period range shown in Figure 9 are accurate to 20\%. Figures 7 and 8 show the corrected dispersion curves and free oscillation periods.

It is interesting to note that much of the discrepancy between the classic models and the data for the low-order fundamental modes is removed by this correction. The agreement of the phase velocity of the Jeffreys model with the observed data is significantly improved. This comparison clearly demonstrates the importance of physical dispersion in the inversion of surface wave and free oscillation data and indicates that modification of these classic models, which is necessary to match the data, may be significantly smaller than has previously been considered. This point was also made by Davies [1967] and Carpenter and Davies [1966]. A recent reinterpretation of the data by Anderson et al. [1976], Hart et al. [1976], and Anderson and Hart [1976b] shows that an earth model can be constructed which satisfies all the corrected surface wave and free oscillation data and that most, if not all, of the discrepancies between body wave and normal modes, such as the 'base line' shift, can be removed.

\section{Conclusion}

Examinations of various absorption models lead us to the conclusion that

$$
C(\omega)=C\left(\omega_{r}\right)\left[1+\frac{1}{\pi Q_{m}} \ln \left(\frac{\omega}{\omega_{r}}\right)\right]
$$

must be used for correcting the effect of physical dispersion arising from anelasticity. In this expression, $C(\omega)$ is the phase velocity of body waves, surface waves, or free oscillations, $\omega$ is the angular frequency of the wave, $\omega_{r}$ is a reference angular frequency, and $Q_{m}$ is the quality factor appropriate for the wave considered. We recommend that a frequency of $1 \mathrm{~Hz}$ be adopted as the standard reference frequency for future work. For body waves, $Q_{m}$ is the path average $Q$, and for surface waves and free oscillations, $Q_{m}$ is the $Q$ of a surface wave or 
mode of frequency $\omega$ under consideration. For surface waves and free oscillations, $C\left(\omega_{r}\right)$ should be understood as the phase velocity at $\omega$ computed by using the elastic moduli at $\omega=\omega_{r}$.

Strictly speaking, this conclusion is not valid for nonlinear models. It is also affected by the values of $Q$ outside the seismic frequency band, which is not very well known. However, if the departure from elasticity is relatively small so that the signal can be approximated by a superposition of propagating harmonic waves, as is usually the case in the earth, the above conclusion is still valid even if the microscopic mechanism of attenuation is nonlinear. The values of $Q$ outside the seismic frequency band affect mainly the absolute value of the phase velocity but do not affect significantly the relative dispersion within the seismic frequency band. Therefore lack of knowledge about $Q$ outside the seismic band does not prevent the use of dispersion relation (15) in surface wave and free oscillation problems.

Since the surface wave $Q$ is relatively low, about 100 at short periods and several hundreds at long periods, the effect of physical dispersion becomes very important in inversion studies.

Acknowledgments. This research was supported by the Advanced Research Projects Agency of the Department of Defense and was monitored by the Air Force Office of Scientific Research under contract F44620-72-C-0078. Contribution 2799 of the Division of Geological and Planetary Sciences, California Institute of Technology, Pasadena, California 91125 .

\section{REFERENCES}

Anderson, D. L., and R. S. Hart, An earth model based on free oscillations and body waves, J. Geophys. Res., 81, 1461-1475, 1976a.

Anderson, D. L., and R. S. Hart, Absorption and the low-velocity zone, Nature, 263, 397-398, $1976 b$.

Anderson, D. L., A. Ben-Menahem, and C. B. Archambeau, A ttenuation of seismic energy in the ppper mantle, J. Geophys. Res., 70, 1441-1448, 1965.

Anderson, D. L., H. Kanamori, R. S. Hart, and H.-P. Liu, The earth as a seismic absorption band, submitted to Science, 1976.

Carpenter, E. W., and D. Davies, Frequency dependent seismic phase velocities: An attempted reconciliation between the Jeffreys/Bullen and the Gutenberg models of the upper mantle, Nature, 212. 134-135, 1966.

Davies, D., On the problem of compatibility of surface wave data, $Q$ and body wave travel times, Geophys. J. Roy. Astron. Soc., 13 . $421-424,1967$

Futterman, W. 1., Dispersive body waves, J. Geophys. Res., 67. $5279-5291,1962$.
Gross, B., Mathematical Structure of the Theories of Viscoelasticity, p. 71, Hermann, Paris, 1953.

Hart, R. S., D. L. Anderson, and H. Kanamori, Shear velocity and density of an attenuating earth, Earth Planet. Sci. Lett., 32, 25-34, 1976.

Jackson, D. D., and D. L. Anderson, Physical mechanisms of seismic wave attenuation, Rev. Geophys. Space Phys., 8, 1-63, 1970.

Jahnke, E., and F. Emde, Tables of Functions, p. 306, Dover, New York, 1945

Jeffreys, H., Damping of $S$ waves, Nature, 208, 675, 1965.

Jefireys, H., Radius of the earth's core, Nature, 215, 1365-1366, 1967.

Kanamori, H., Velocity and $Q$ of mantle waves, Phys. Earth Planet. Interiors, 2, 259-275, 1970.

Knopoff, L., Q. Rev. Geophys. Space Phys., 2, 625-660, 1964.

Knopoff, L., and G. J. F. MacDonald, Attenuation of small amplitude stress waves in solids, Rev. Mod. Phys., 30. 1178-1192, 1958.

Kogan, S. Ya., A brief review of seismic wave absorption theories, 2 , Izv. Acad. Sci. USSR Phys. Solid Earth, Engl. Transl., no. II, 678-683, 1966.

Kolsky, H., The propagation of stress pulses in visco-elastic solids, Phil. Mag., 1(8), 693-710, 1956.

Lamb, G. L., Jr., The attenuation of waves in a dispersive medium, $J$. Geophys. Res., 67, 5273-5277, 1962.

Liv, H.-P., D. L. Anderson, and H. Kanamori, Velocity dispersion due to anelasticity: Implications for seismology and mantle composition, Geophys. J. Roy. Astron. Soc., 47, 41-58, 1976.

Lomnitz, C., Linear dissipation in solıds, J. Appl. Phys., 28, 201-205, 1957.

Mason, W. P., Internal friction mechanism that produces an attenuation in the earth's crust proportional to the frequency, $J$. Geophys. Res., 74, 4963-4966, 1969.

Morse, P. M., and H. Feshbach, Methods of Theoretical Physics, part 1, McGraw-Hill, New York, 1953.

Nowick, A. S., and B. S. Berry, Lognormal distribution function for describing anelastic and other relaxation processes, $1,2, I B M J$. Res. Develop., 5, 297-320, 1961.

Randall, M. J., Attenuative dispersion and frequency shifts of the earth's free oscillations, Phys. Earth Planet. Interiors, 12, 1-4, 1976.

Savage, J. C., Attenuation of elastic waves in granular mediums, $J$. Geophys. Res., 70, 3935-3942, 1965.

Savage, J. C., Anelastic degradation of acoustic pulses in rockComments, Phys. Earth Planet. Interiors, 11, 284-285, 1976.

Stacey, F. D., et al., A nelastic damping of acoustic and seismic pulses, Geophys. Surv., 2, 133-151, 1975.

Strick, E., The determination of $Q$, dynamic viscosity and transient creep curves from wave propagation measurements, Geophys. $J$. Roy. Astron. Soc., 13. 197-218, 1967.

(Received August 23, 1976; accepted August 31, 1976.) 